\title{
Virtual Reality Education Modules for Digital Manufacturing Instruction
}

\section{Dr. Magesh Chandramouli, Purdue University Northwest}

Dr. Chandramouli is an Associate Professor of Computer Graphics Technology in Purdue University Northwest. He has been invited to deliver keynote speeches and lectures in various countries and universities around the world. Formerly a Frederick Andrews Fellow at Purdue University, West Lafayette, he completed his doctoral studies from the Department of Computer Graphics Technology. He has received federal, regional, and international grants for his work.

\section{Dr. Ge Jin, Purdue University Northwest}

Ge Jin, D.Sc, is currently an associate professor in the Department of Computer Information Technology and Graphics at the Purdue University Calumet. He teaches computer game development, computer graphics and animation, as well as computer information technology courses at the undergraduate and graduate levels. Prior to joining Purdue University Calumet, he was a postdoctoral research scientist at the George Washington University, Department of Computer Science. Professor Jin holds a B.S. in Computer Science from Peking University, China, and an M.S. in Computer Science from Seoul National University, South Korea. He earned his Doctor of Science degree in Computer Science with a concentration in computer graphics from the George Washington University. His research spans the fields of computer graphics, virtual reality, computer animation, medical visualization, and educational game development. He is a member of the ACM SIGGRAPH, ASEE, and International Society of Virtual Rehabilitation.

\section{Mr. Justin David Heffron, Purdue University Northwest \\ Dr. Ismail Fidan, Tennessee Technological University}

Currently, Dr. Fidan serves as a Professor of the Department of Manufacturing and Engineering Technology at Tennessee Technological University. His research and teaching interests are in additive manufacturing, electronics manufacturing, distance learning, and STEM education. Dr. Fidan is a member and active participant of SME, ASEE, ABET, ASME, and IEEE. He is also the Associate Editor of IEEE Transactions on Components, Packaging, and Manufacturing Technology and International Journal of Rapid Manufacturing.

\section{Dr. Mel Cossette, Edmonds Community College}

Mel Cosette is the executive director and principal investigator for the National Science Foundationfunded National Resource Center for Material Technology Education (MatEd) housed at Edmonds Community College in Lynnwood, Wash. Mel has over 20 years of experience in manufacturing education and has developed technician-training programs for industry and educational institutions. She serves on numerous committees and national boards, and worked in various industries prior to holding administrative positions in the community and technical college system.

\section{Cheryl A. Welsch, Eastern Iowa Community College}

Cheryl Welsch is Lead Developer for Information Technology, Virtual Reality and Business Programs in the Eastern Iowa Community College District. She oversees curriculum design, staff recruitment and development, and external relations. Ms. Welsch earned her M.B.A. from Western Governors University in Information Technology Management and her B.A. from the State University of New York - Cobleskill in Business with a concentration in Information Systems. She has earned the designation Certified Information Security Manager (CISM) and is a member of ISACA, formerly known as the Information Systems Audit and Control Association. She has spoken on information security at numerous professional associations, is the technical director for a global investment publication.

Wayne Merrell 


\title{
Virtual Reality Education Modules for Digital Manufacturing Instruction
}

\begin{abstract}
There is an imminent need to remedy the 'skills gaps' in the digital manufacturing (DM) sector as evident from the Bureau of Labor Statistics projections pointing to a decline in traditional manufacturing jobs accompanied by marked growth in digital- and computer-driven manufacturing jobs. With proven advantages such as cost benefits, material conservation, minimized labor, and enhanced precision, manufacturing industries worldwide are adapting to digital manufacturing standards on a large scale. In an effort to remedy the lack of well-defined DM career pathways and instructional framework, our NSF ATE (Advanced Technological Education) project MANEUVER (Manufacturing Education Using Virtual Environment Resources) is developing an innovative pedagogical approach using virtual reality (VR). This multimodal VR framework DM instruction targeted at 2-year and 4-year manufacturing programs, facilitates the development of VR modules for multiple modes such as desktop VR, Augmented VR, and Immersive VR. The advantages of the virtual reality framework for digital manufacturing education include: significant cost reduction, reduction in equipment and maintenance costs, ability to pre-visualize the product before manufacturing. This paper introduces the design and development process of VR education tool to simulate three different additive manufacturing machines, e.g., LutzBot ${ }^{\mathrm{TM}}$, FormLabs ${ }^{\mathrm{TM}}$, and UPrint ${ }^{\mathrm{TM}}$ and different 3D printing technologies e.g., fused deposition modeling, and selective laser sintering to allow the students experience the materials and equipment needed to create the same part using different types of equipment and different types of technology.
\end{abstract}

Keywords - Digital Manufacturing, Virtual Reality, Desktop VR, Augmented VR

\section{Background and Related Work}

DM offers advantages such as cost benefits, material conservation, minimized labor, and enhanced precision. 'Digital manufacturing uses a 'digital thread' to link every phase in the manufacturing lifecycle, from early stages such as design through to sale and service. The goal of digital manufacturing is to provide manufacturers with better insight at critical decision points to avoid costly errors, gain efficiencies and be able to respond to customer and market demands in a more agile manner" (Digital Manufacturing, 2016). At present, there is no clearly defined career or educational pathway for preparing technicians with DM skill set(s). Hence, there is a clear and imminent need to educate and train our future workforce with the skills required to avail the opportunities from the DM boom. An economic and efficient way to accomplish this will be to train the trainers that deliver technician education. Advances in DM technologies have revolutionized key aspects of manufacturing including design, development, testing, etc.

The Deloitte Review (GMCI, 2016) points out a concern that is relevant in this context: “A skills gap is the US manufacturing sector's Achilles' heel, with nearly 3.5 million jobs at stake over the next decade. It is no longer a short-term issue of filling current hard-to-fill open positions, or one that can reasonably be expected to be solved in time by government policy-makers. " The following excerpt from EMSI (2015) is especially relevant from the perspective of "training-thetrainers': "According to EMSI quarter 2 data it is projected that between 2016-2025 there will 
be over 21,000 new high-tech manufacturing/computer based jobs within Chicago and Northwest Indiana, region 1. Providing highly specialized digital manufacturing "train the trainer" style professional development will aid in creating a fluid pipeline of entry-level technicians to fill the large number of projected future opportunities."

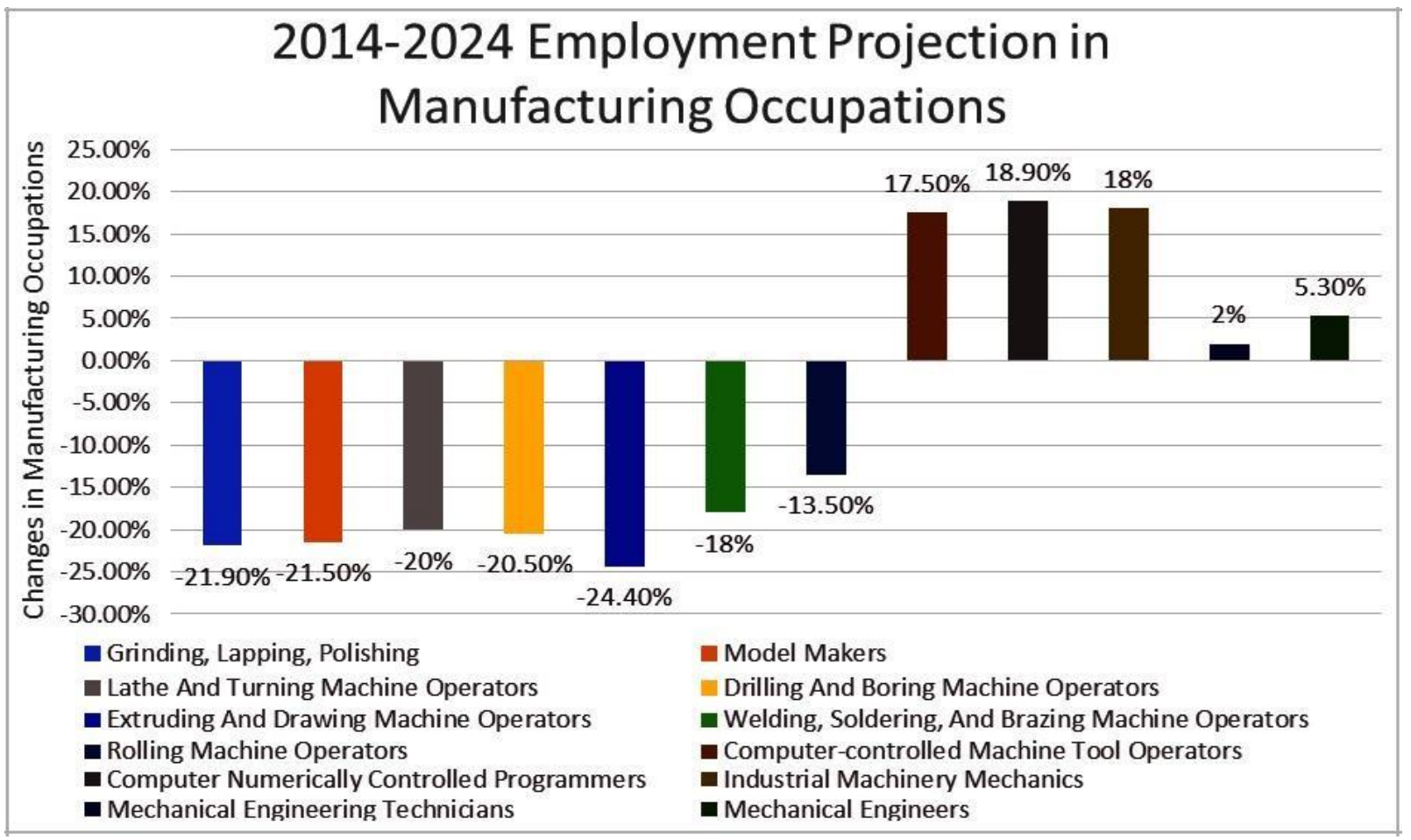

Figure 1: BLS employment projection from 2014 to 2024 in manufacturing sector. (BLS, 2014)

As seen in Figure 1 (BLS, 2014), the employment projections from 2014 to 2024 show a decline in conventional manufacturing jobs accompanied by marked growth in digital- and computerdriven manufacturing jobs. The Bureau of Labor Statistics (BLS, 2014) has projected a 7\% decrease in the traditional manufacturing workforce (814,100 jobs) from 2014 to 2024. Meanwhile, the U.S. digital manufacturing industry is estimated to grow by $7.55 \%$ from 2015 to 2019 (Research and Markets, 2015).

An insightful observation made more than a decade ago about barriers to learning is still relevant to our current challenge pertaining to digital manufacturing instruction. Wohlers Report (Wohlers, 2001) stated, "the most significant barrier to realizing new applications and powerful benefits is the tendency to resist change. Established processes and procedures (in Rapid Prototyping) are difficult to displace." This may also be equally applicable to academic scenarios involving digital manufacturing instruction. Academic institutions as well as instructors may resist adapting to the new technologies because of: (1) The unwillingness to depart from traditional manufacturing concepts; and (2) The lack of awareness of the benefits of digital manufacturing.

With due consideration to the issues and concerns discussed and considering the imminent need for well-trained technicians to match the demands of the manufacturing industry, interactive VR- 
based tools can be quite effective in stimulating interest and reducing cognitive load (Chandramouli \& Heffron, 2015, Dias, Sousa, Parracho, Cardoso, Monteiro, \& Santos, 2014).

\section{Methodology}

The overall goal for MANUEVER is to: Develop an innovative multi-modal VR framework for digital manufacturing instruction. This multi-modal VR will allow customization for access via (1) desktop VR (dVR); (2) augmented VR (aVR); and (3) immersive VR (iVR), thereby targeting a broader audience with diverse budgetary constraints. Each of these modes has its own advantages and disadvantages. While iVR offers high-fidelity immersion and navigation, it has a very high cost and space implications. On the other hand, $d V R$ is quite affordable but lacks the immersive functionalities of iVR. Not all interactive DM applications/modules require the use of immersive systems (Chandramouli et al., 2014). Training programs can opt for lesser levels of immersion with aVR/dVR at lower cost to accomplish similar goals. The VR framework design includes 3 components as illustrated in Figure.2:

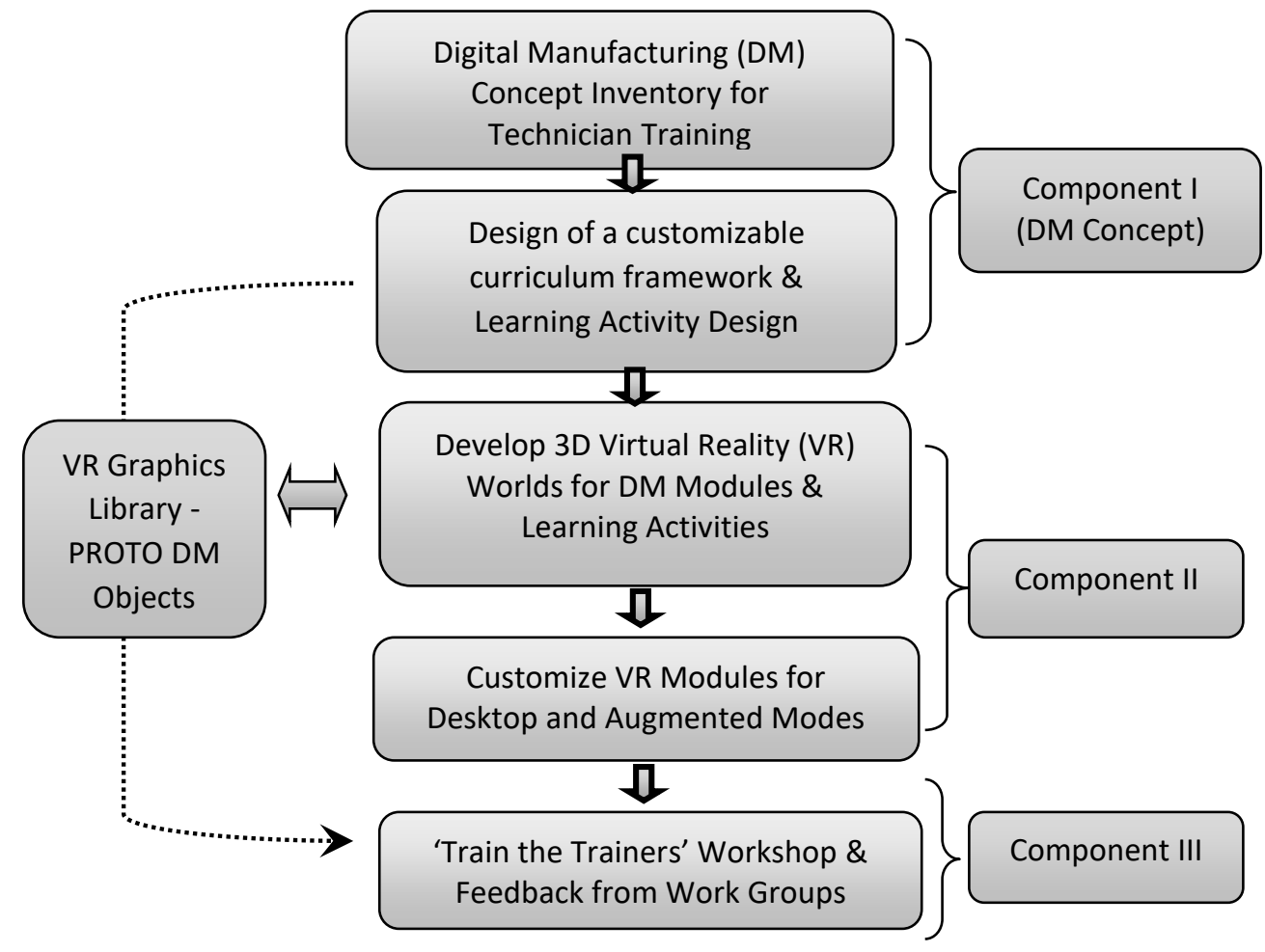

Figure 2. Basic Framework for Module Generation

3D Virtual worlds can be visualized using a wide range of display devices including desktop VR, CAVE, HMD, and augmented VR etc. Each of the aforementioned platforms offers unique functionalities which may or may not be available in others. For instance, CAVEs offer high end fidelity, immersion and navigation. However, CAVEs involve large spaces and cumbersome installation procedures. CAVEs also entails significance caused with respect to installation, operation and maintenance. On the other hand, dVR (desktop Virtual Reality) 
systems offer good functionalities for online dissemination and dynamic interaction in realtime. However, the level of immersion and navigation offered by such systems remains questionable. An ideal system would incorporate all desired characteristics in a VR platform including immersion, navigation, dissemination, fidelity and portability. The last mentioned characteristic, portability is a key area where significant and concerted efforts are needed. The advantages of a pVR (portable Virtual Reality) system are two-fold. While a VR scene built for a CAVE system can be modified for display on a dVR, this would come at the cost of compromising immersion and navigation capabilities. A meticulously designed VR scene with emphasis on immersion and navigation would lose much of the intended benefits when shown to a client on an alternative UI/platform devoid of I/N (Immersion/Navigation) capabilities. For instance, a dVR system can neither appropriately capture nor display a visualization originally designed for a CAVE system in its entirety; however, with laptops used for dVR, portability is a notable advantage while $\mathrm{I} / \mathrm{N}$ are compromised. Hence in this paper we design and implement a pdVR (portable desktop Virtual Reality) system that represents an optimal trade off while offering portability. Also, this system, to a reasonable extent facilitates Immersion/Navigation/Interaction (I/N/I) (Figure.3).

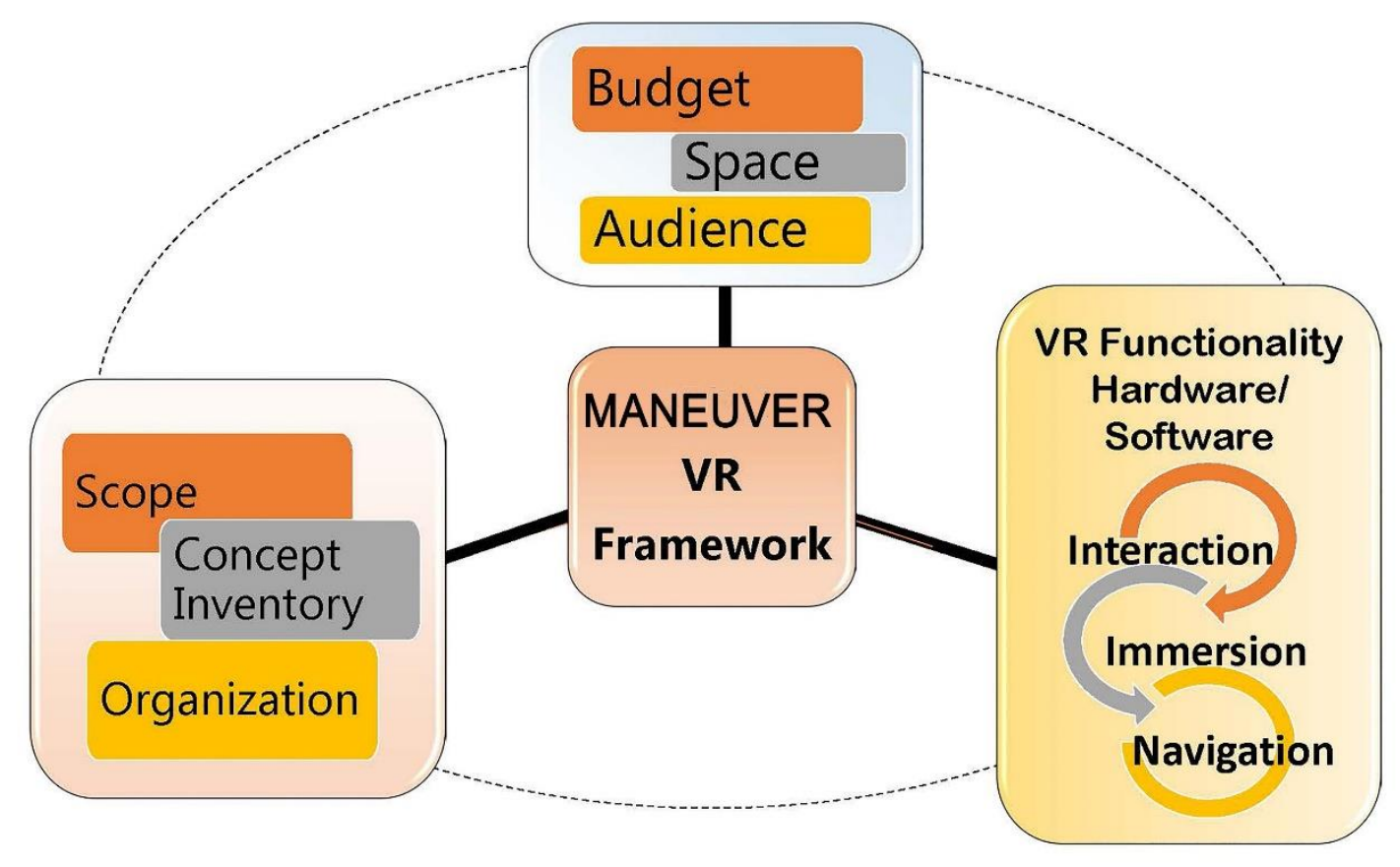

Figure.3. MANEUVER Virtual Framework: Factors, Scope, \& Considerations

\section{Identification of VR training topics for digital manufacturing education}

VR, as an instructional training tool for Digital Manufacturing presents the potentials of promoting student interest in the training/learning process. VR is a proven tool that can engage learners effectively and the enhanced engagement can be attained by actively involving the participant or trainee in the process (Toth, Ludvico, \& Morrow, 2014, Chandramouli, Zahraee, \& Winer, 2014, Jin \& Nakayama, 2013, Jen, Taha, \& Vui, 2008). Laboratory exercises in manufacturing curriculum are not always able to meet the demands of the advanced 
manufacturing need in their conventional form with the traditional experiments. Virtual laboratories, on the other hand, can be built to simulate various devices and equipment and programmed to allow interaction with these devices to understand processes, assemble components, and perform trial-and-error procedures without risking equipment damage. The virtual laboratories and training modules, once built, can be replicated for use on multiple machines without any additional costs as this involves just copying the required software and training modules to other machines. Furthermore, during the training for manufacturing processes, VR tools serve as a viable alternative offering a cost and material-efficient solution by replacing the need for actual physical materials. Although virtual laboratories may not entirely replace physical laboratories, VR practices can lead to considerable cost and time savings by limiting the use of physical lab/equipment to only when actual implementation or manufacturing is required. A state-of-the-art VR-based curriculum with the above benefits will be designed to educate and train skilled technicians. The curriculum design from a digital manufacturing perspective will include the following: 1) A VR laboratory capable of delivering digital manufacturing conceptual (theoretical) and practical training using carefully designed training modules, 2) Extensible VR modules designed to support immersion, navigation, and interaction, 3) A comprehensive set of replicable DM training exercises that can be performed by students/ trainees with minimal supervision.

To identify digital manufacturing curricular inputs, a survey was conducted involving all authors to create digital manufacturing concept inventory, from which key topics were identified for VR module generation. Based on initial discussion among authors, seven areas in digital manufacturing were selected for the survey. The seven areas are: Pre-Visualization, Virtual Product Assembly, Fused Deposition Modeling (FDM), Product Simulation, Reverse Engineering, Additive Manufacturing, and Direct Digital Manufacturing (DDM). The core survey questions and results are shown in Figure. 4. The survey results indicate that Additive Manufacturing, Virtual Product Assembly, and Direct Digital Manufacturing are three areas with high priority.
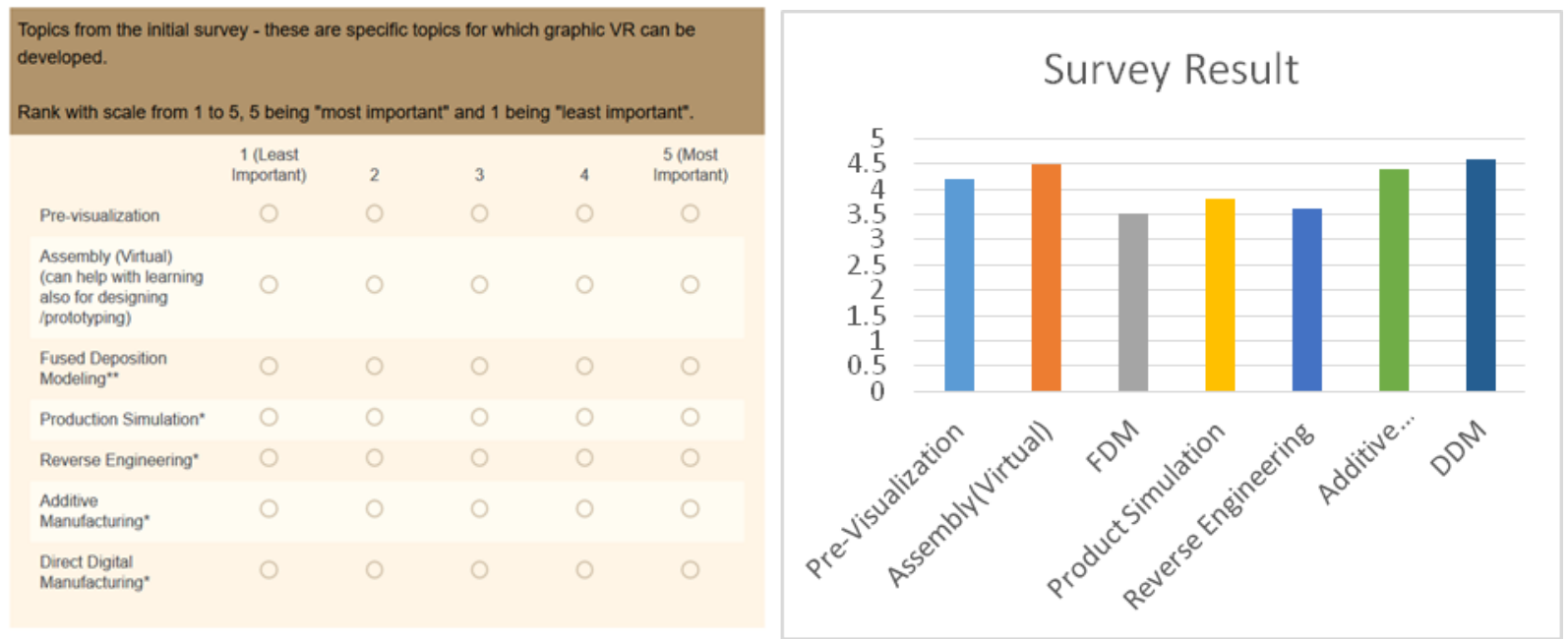

Figure.4. Survey Questions and Results to Identify VR training Topics 


\section{Simultaneous module development for both Augmented and Desktop VR Modes}

Before proceeding further, it is important to briefly look at the concepts of additive and subtractive manufacturing to understand why the modules have been generated. Subtractive manufacturing involves creating objects by removing (or subtracting) material from a solid block of material (e.g. metal). Typically, machinery and components manufactured with a CNC Machine fall under this category. This cutting is actually done in the 3D space $(\mathrm{x}, \mathrm{y}, \mathrm{z})$ and numerous advances have been made in $\mathrm{CNC}$ machine technology over the years that have greatly enhanced the processes. On the other hand, with additive manufacturing objects are built by consecutively placing material in the form of layers (one on top of the other) so as to create a specific shape (Figure.5). Depending on the actual product and industry, these steps may change but these represent some of the basic steps generally followed in additive manufacturing processes.

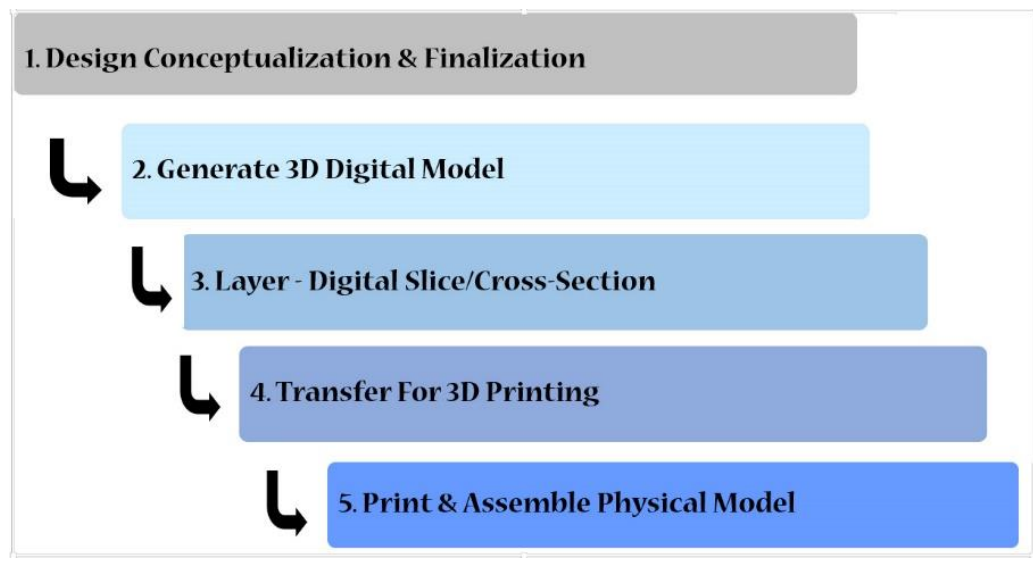

Figure 5. Basic Interaction Exercises within the 3D World \& Importing 3D STL Meshes When viewed with standard desktop virtual reality on standard web browsers, additional lowcost devices such as TouchPad and Stylus Pen can be used to enhance interactions and navigation functionalities. (Figure. 6)

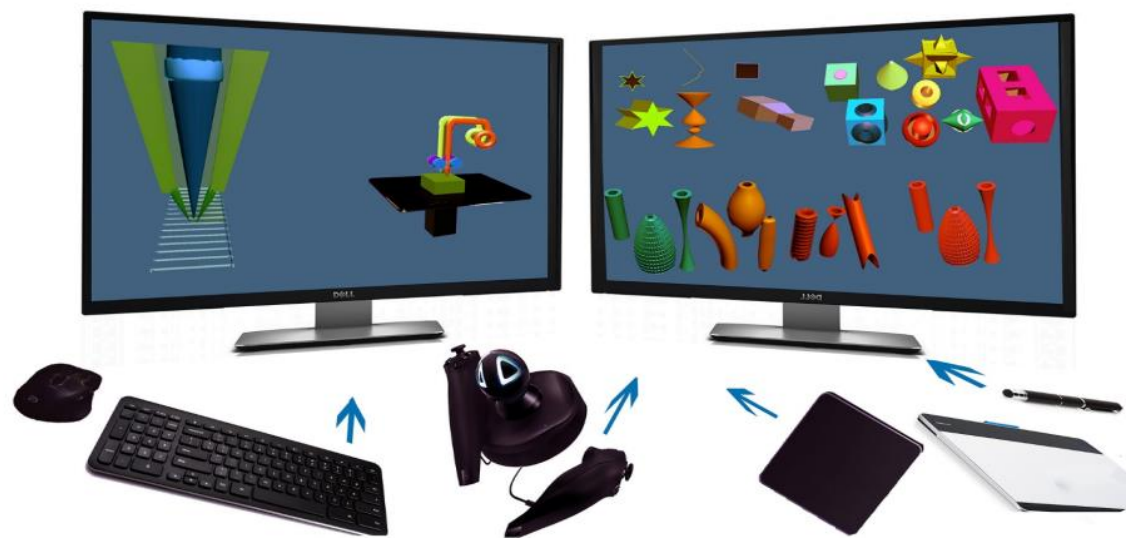

Figure. 6. Interaction with 3D World Using Standard Affordable I/O devices 
The VR training modules that will be developed for digital manufacturing education are: 1 . Additive manufacturing product comparison, 2. Direct digital manufacturing with computer numerical control (CNC) machine, and 3. Virtual product assembly. These topics were selected based on the survey and study described in the previous section.

Comparing different additive manufacturing products and technologies will provide students with an opportunity to compare a designated printed part by additive manufacturing machines available from three different companies, e.g., LutzBot ${ }^{\mathrm{TM}}$, FormLabs ${ }^{\mathrm{TM}}$, and UPrint ${ }^{\mathrm{TM}}$. The 3D printing technologies that employ these machines are Fused Deposition Modeling (FDM) technology (LutzBot ${ }^{\mathrm{TM}}$, and UPrint ${ }^{\mathrm{TM}}$ ) and Stereolithography technology (FormLabs ${ }^{\mathrm{TM}}$ ). The VR learning modules simulating different additive manufacturing technologies will provide students with materials and equipment needed to create the same part on three different AM machines. Students can utilize three different types of equipment to understand that the same technology can be demonstrated in different processes and still produce a similar product. Figure. 7 below shows the simulation of three popular 3D printing devices namely UPrint ${ }^{\mathrm{TM}}$, $\operatorname{LutzBot}^{\mathrm{TM}}$, and FormLabs $^{\mathrm{TM}}$, from left to right.

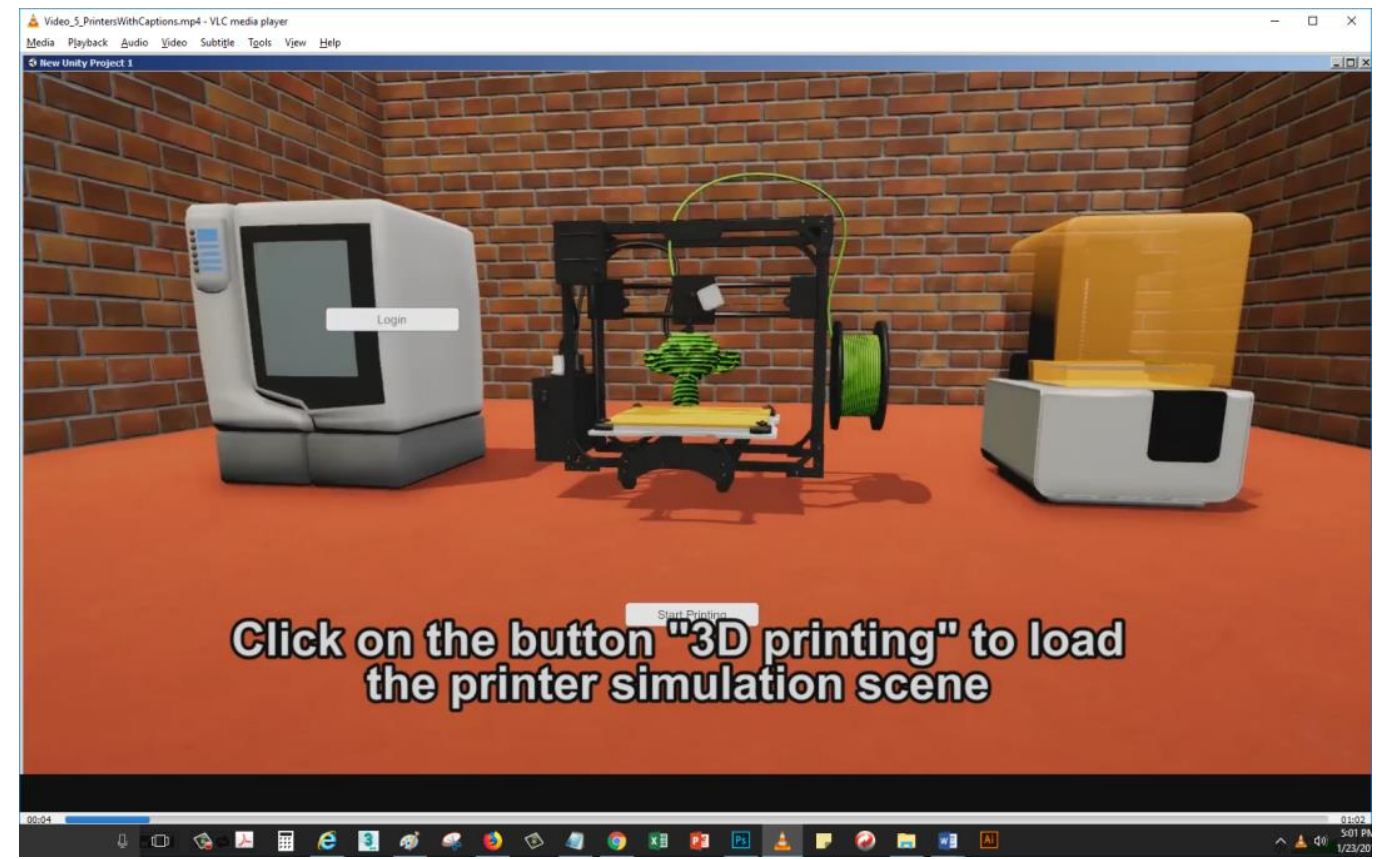

Figure 7. VR Simulation of three 3D printing machines (UPrint ${ }^{\mathrm{TM}}$, LutzBot ${ }^{\mathrm{TM}}$, FormLabs ${ }^{\mathrm{TM}}$ )

This section discusses the simulation of one major subtractive digital manufacturing machinery: computer numerical control (CNC) machine. Both additive manufacturing simulation and $\mathrm{CNC}$ simulation were developed in Unity3D game engine. To simulate the virtual CNC milling process, three game development techniques were employed including a NavMesh system, a mesh collider and a Mesh voxelizer. The mesh voxelizer will pre-process the final 3D model of product to be manufactured, and subdivide the mesh into thousands of small cubes. The mesh collider will determine if the drill head touches individual voxelized cube based on the drill head movement. If the drill head touches the cube, the touched cube will be removed from the scene, thus simulating the subtractive manufacturing effect. The NavMesh system utilizes built-in 
Artificial Intelligent (AI) pathfinding system. The final output mesh would be marked as a nonwalkable agent, meaning the moving milling head would avoid touching it. The remaining area to be carved out would then be procedurally baked for NavMesh movement. Alternatively, the final mesh in the center can be defined as a step, meaning the AI drill bit would move over it while moving up vertically. This would give the appearance of the drill bit excluding the mesh while still moving in a normalized and predictable pattern. One possible movement path would be to have the drill bit move left and right in rows while moving up each time that a row is completed. The destination point of the NavMesh agent can be dynamically set via coding.

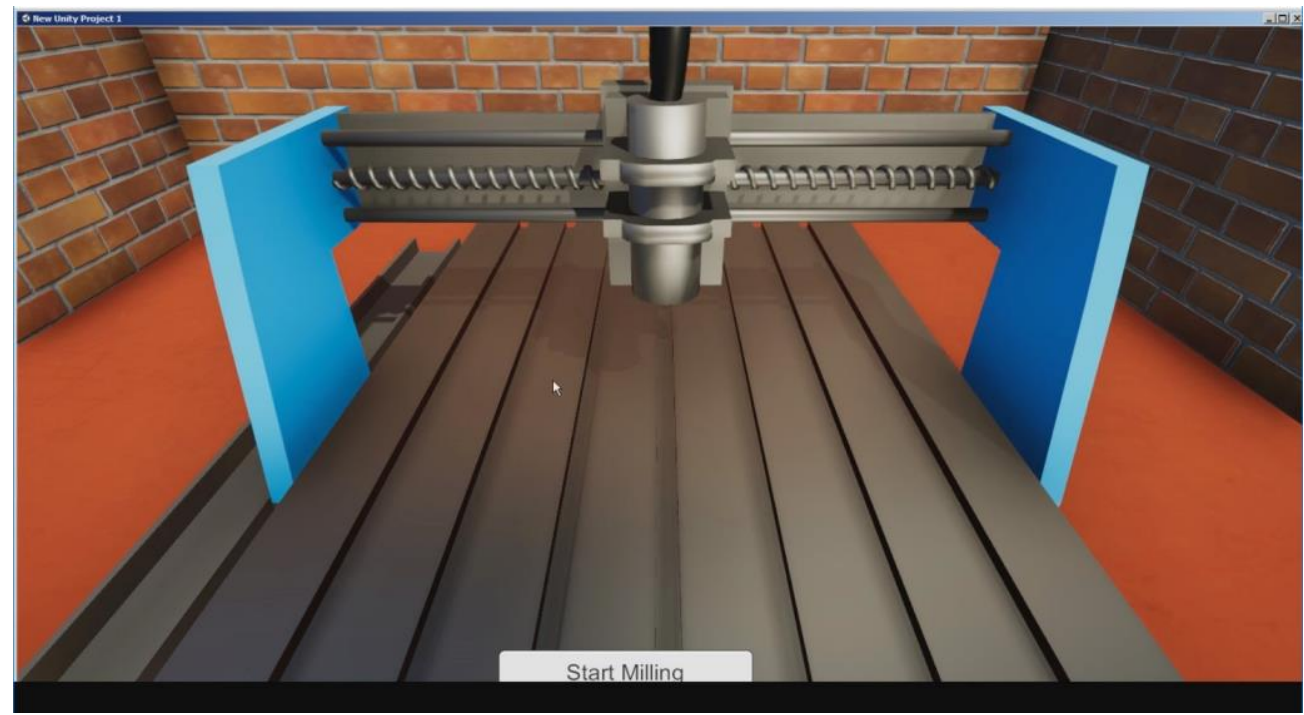

Figure 8. Virtual CNC Milling Simulation (Unity3D Game Engine)

The third topic for VR training module is virtual product assembly. Digitally manufactured parts from either additive manufacturing or subtractive manufacturing need to be assembled to become a product. Currently, the virtual assembly process was implemented using simple 3D geometric shapes to focus on the user interaction with virtual 3D parts. A user can also import digitally manufactured 3D part file to be assembled with another part. Figure. 9 shows three main functionalities of virtual product assembly process. A user can use the 'W', 'A', 'S', and 'D' keyboard key to move and navigate a 3D scene. A keyboard shortcut key ' $\mathrm{I}$ ' can be used to import the mesh that represents a digitally manufactured 3D part. Keyboard shortcut key 'E' will be used to pick up and move a 3D part to a new location to assemble with other 3D parts.
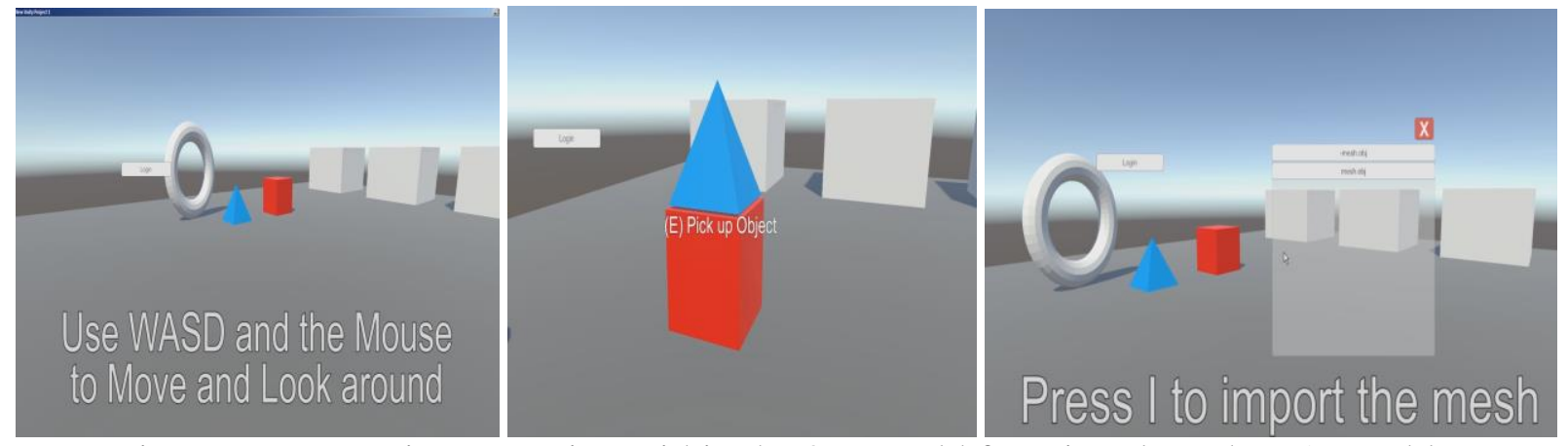

Figure.9. Basic Interaction within the 3D World for Virtual Product Assembly 
The above VR training modules are presented in the form of a game-like interface that makes the interaction more fun-based and reduces the cognitive load. Unity/WebGL platform is used for the virtual simulation of digital manufacturing processes. To facilitate visualization using normal desktop computers, simulation from a local host and the 'MANEUVER' database was employed. This way, educational institutions including community colleges who may not have the financial resources, can still view and interact with the digital manufacturing (DM) modules using desktop computers that are typically available in most labs. Also, this option avoids having to actually go through the tedious process of requiring educational institutions to have a local host server up and running. Figure. 10 below demonstrates this server simulation process and performing basic 3D interaction exercises. Once the setup is completed, the modules can be accessed just as a webpage can be accessed from a local server.
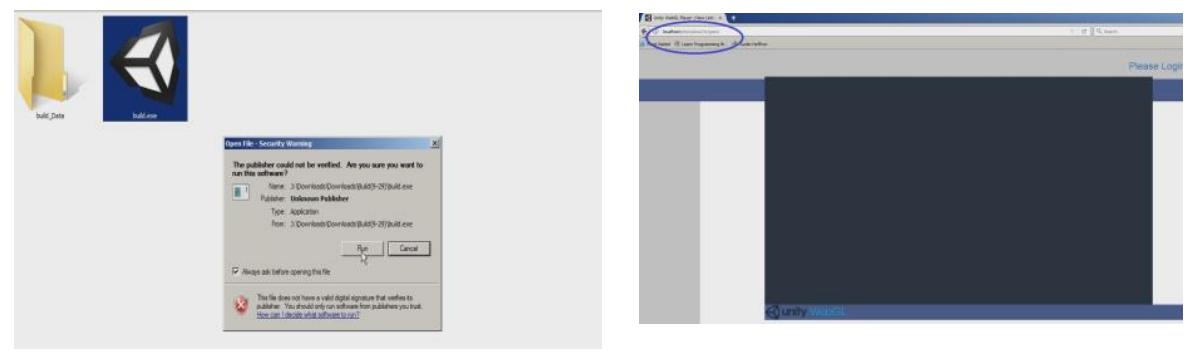

Figure.10. Local Server Simulation and Accessing VR Modules Using Web Browser

\section{Results and Discussion}

This section presents the development results of virtual reality training modules for digital manufacturing and discusses the simulation of one major subtractive manufacturing example and 3 popular examples of additive manufacturing machines.

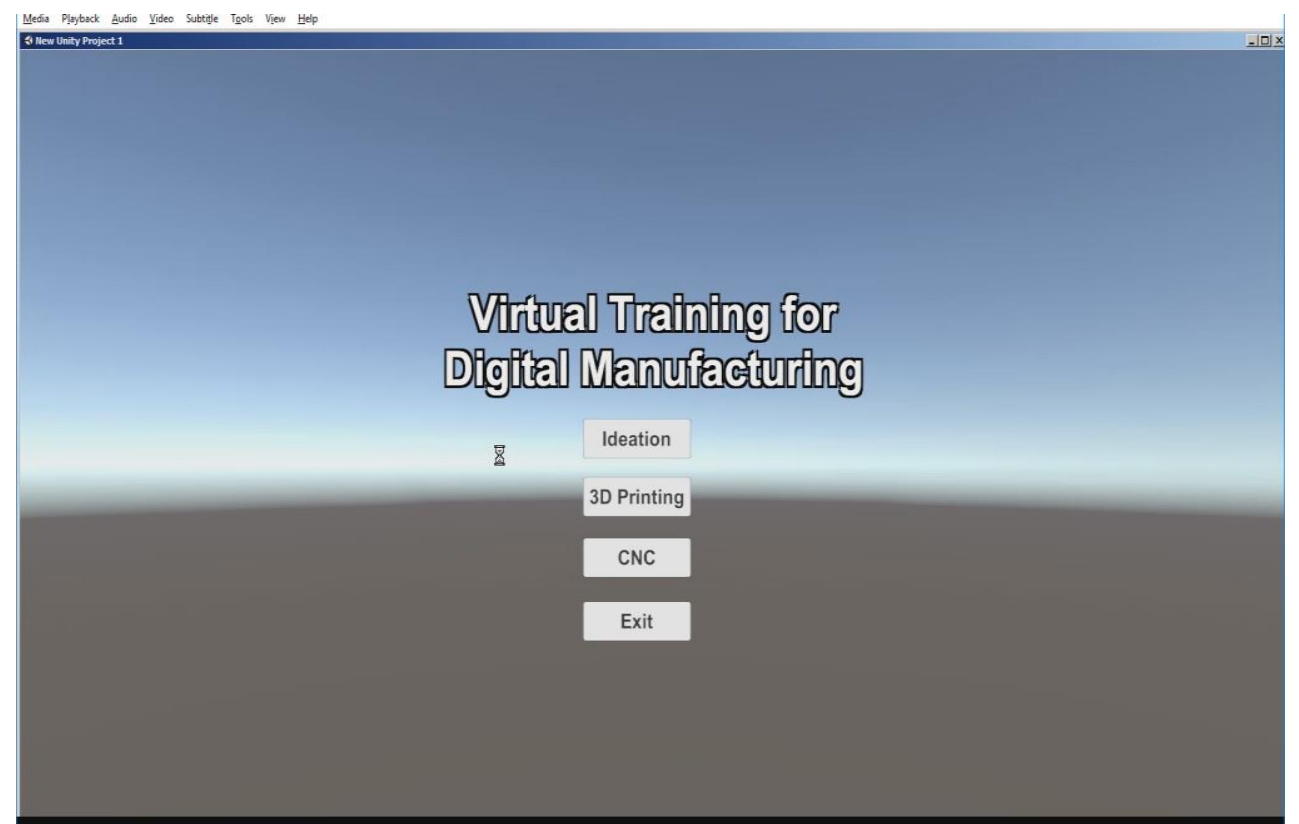

Figure 11. Main Menu of Virtual Reality Training Modules for Digital Manufacturing Education 
Figure. 11 shows the main user interface of VR learning modules for digital manufacturing instruction. The "Ideation" button links to virtual product assembly module, the "3D Printing" button links to three Additive Manufacturing comparison module and the "CNC" button links to computer numerical control machine module.

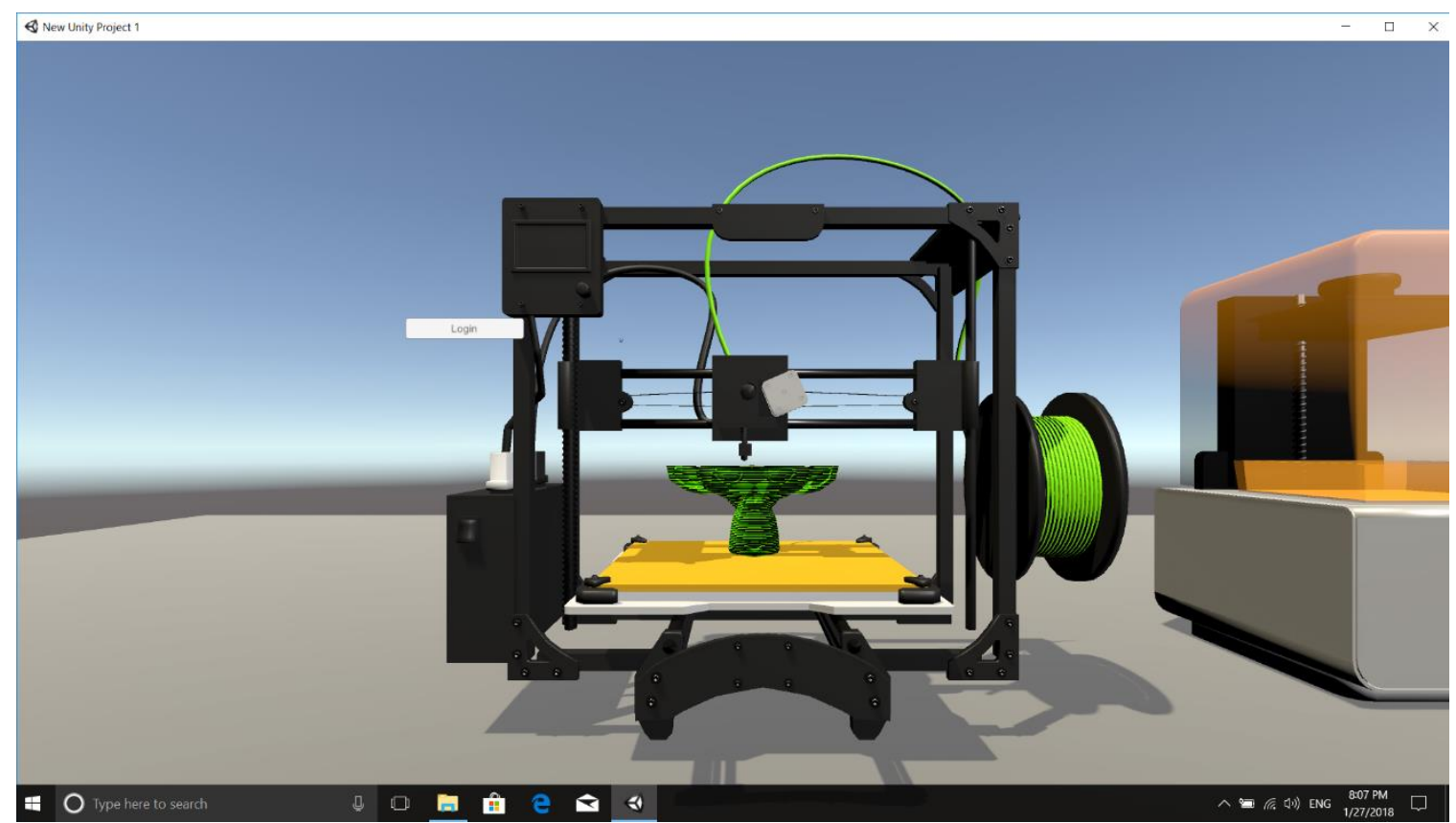

Figure 12. Simulation of Fused Deposition Modeling Technology with LutzBot ${ }^{\mathrm{TM}}$ 3D Printer

For the Additive Manufacturing comparison module, we will present the simulation results of two distinct 3D printing technologies: FDM and Stereolithography. For the FDM technology simulation, a pre-imported mesh was used to test the functionality of the 3D printer system with the LutzBot ${ }^{\mathrm{TM}}$ 3D printer. However, it is required that users be able to import their own models to see how the process works. In order to accomplish this, the same basic system from importing in the ideation scene was used. In this system, a model is loaded from the local directory and placed in an empty game object in the scene. A new empty game object prefab was created for the printer scene since this required a different material than the models imported in the previous scene. The simulation result of fused deposition modeling with LutzBot ${ }^{\mathrm{TM}} 3 \mathrm{D}$ printer is shown in Figure. 12.

To simulate stereolithography technology, The FormLabs ${ }^{\text {TM }} 3 \mathrm{D}$ printer requires a new script for handling the motion of the printer. Unlike the FDM based 3D printer (and other standard filament printers) the FormLabs ${ }^{\mathrm{TM}} 3 \mathrm{D}$ printer creates material with PLA resin rather than strings of plastic filament. The motion consists of a printer head attached to the printer mesh which gradually pulls the mesh out of the vat of liquid. A scraper moves along the liquid to level it when the printer head is raised and a laser solidifies the material when the head is down and in the correct position. In order to create the motion of parts stopping and waiting for other parts, a co-routine was used. Co-routines are functions which run parallel to the main program and do not stop the functioning of the main program while loops are running. One can include a return inside of loops to cause only one iteration to run each frame. It also has return functions for waiting where after a defined number of seconds, the co-routine will resume running. Two 
different routines are used for the printer head and scraper. The scraper will only move while the head is moved up and is waiting to move down again. The laser motion runs when the

FormLabs ${ }^{\mathrm{TM}} 3 \mathrm{D}$ printer head is down. It generates a random position within the printer bounds to simulate the rapid jumping around of the real printer. A co-routine is run with wait functions, albeit very fast ones, to change the position every tenth of a second (6 frames) rather than every frame. Figure. 13 shows the simulation result of stereolithography technology with FormLabs ${ }^{\mathrm{TM}}$ $3 \mathrm{D}$ printer.

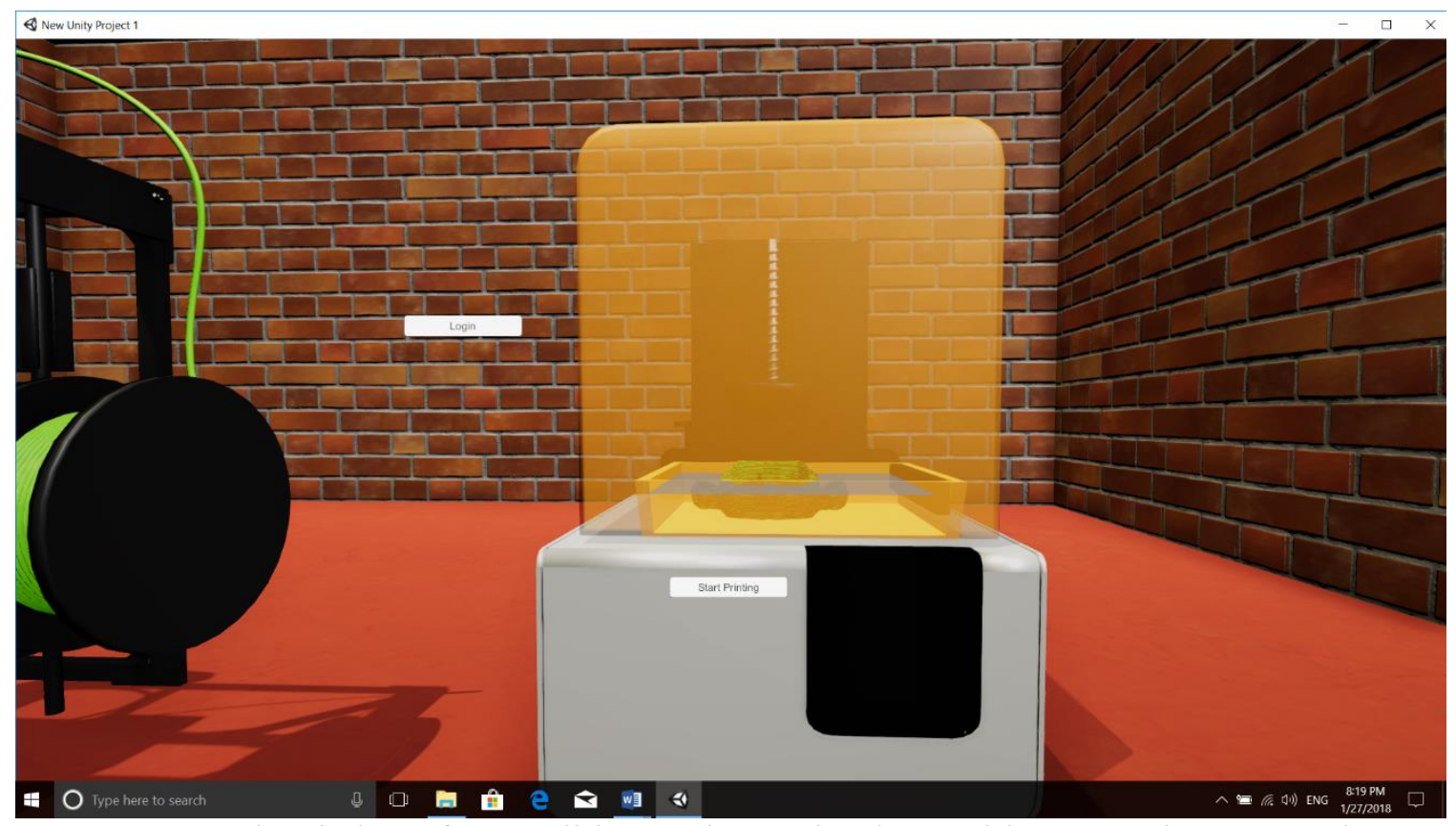

Figure 13. Simulation of Stereolithography Technology with FormLabs ${ }^{\text {TM }}$ 3D Printer

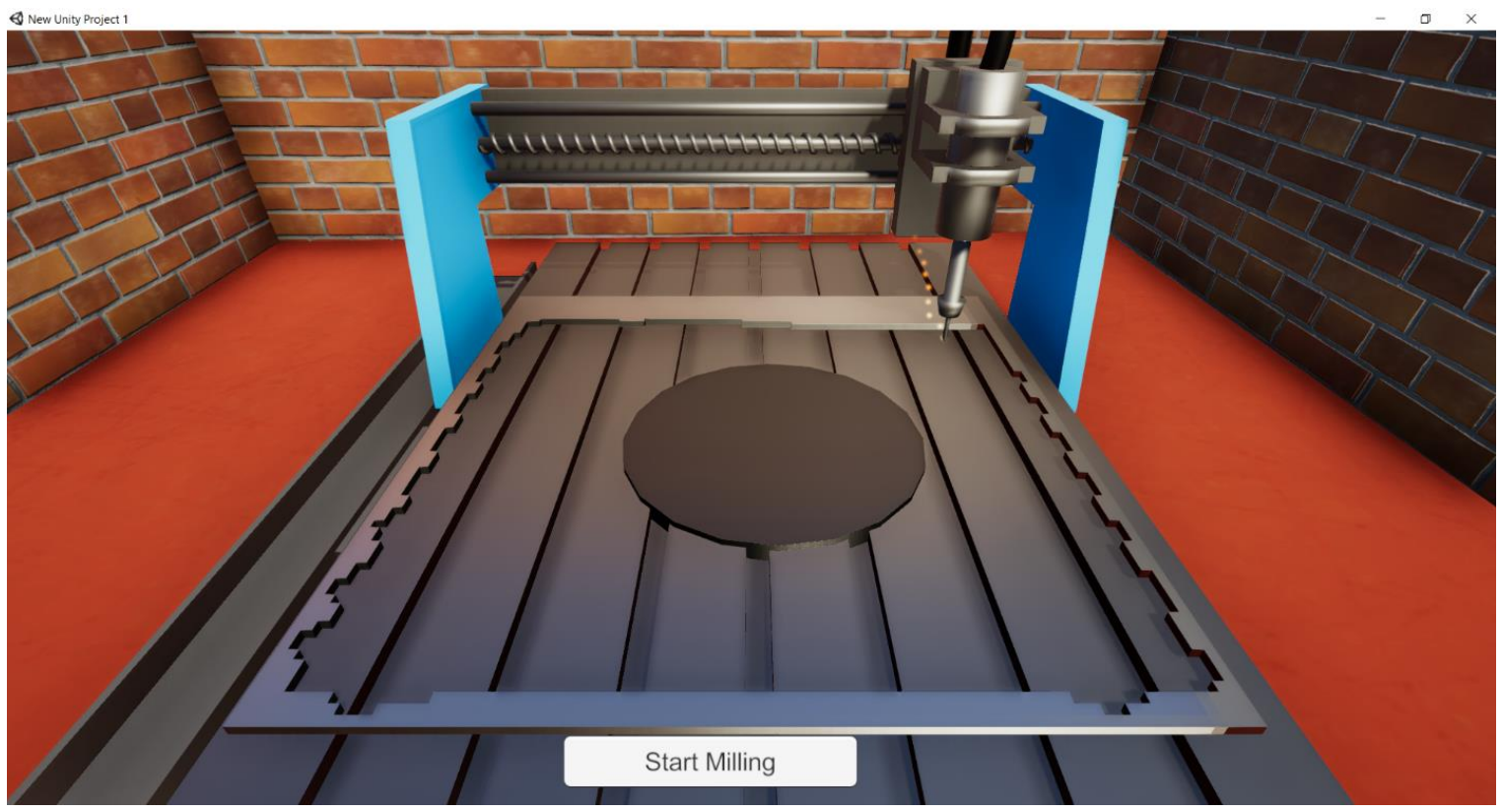

Figure 14. Simulation of Computer Numerical Control Machinery 
Figure. 14 shows the simulation result of CNC milling process using Unity3D NavMesh system and Mesh Voxelizer script. The delivery of developed virtual reality framework for digital manufacturing instruction is scheduled for summer 2018. Hence, the detailed results from the workshop will be collected and the comprehensive evaluation will be published subsequently.

\section{Limitations and Considerations}

This work shows the work accomplished in the first two quarters of the year.1 and the workshop is still to be conducted as it is scheduled for the upcoming summer. The workshop will be conducted at the participating universities and it will involve imparting training to digital manufacturing instructors (train-the trainers approach). Detailed feedback will be obtained from the participants and comprehensive quantitative/qualitative evaluations will be carried out. These will be presented in conferences and published as well. Furthermore, focus groups of participants will be included during the workshops to actively engage the instructors and solicit their critiques on methods implemented thus far and for future enhancement.

\section{Conclusion}

This effort creates a virtual reality framework to digital manufacturing-pathway for small manufacturing organizations as well as those companies and educational institutions that cannot afford to establish a lab with physical equipment. VR is an additional way to connect students with potential careers, specifically within additive manufacturing. This project employs an innovative pedagogical approach using multi-modal VR for DM instruction specifically targeted at 2-year Associate degree programs. Currently, there is no clearly defined career and educational pathway(s) for preparing entry-level technicians in DM. The faculty partners will capitalize on prior experience in VR and manufacturing projects to design user-friendly DM instructional modules. Based on successful VR modules developed/presented/published earlier by the faculty partners, this project aims to build a larger comprehensive framework for instructors and students to overcome the inherent 'resistance to change'. Especially, the innovative multi-modal design will allow customization for access via: 1) desktop VR (dVR); 2) augmented VR (aVR); 3) high-fidelity immersive VR (iVR), thereby targeting a broader audience with diverse budgetary constraints. VR education modules for Digital Manufacturing can be used for instruction at 2-year and 4-year programs to serve as a collaborative product design/development tool to facilitate project-based learning (PBL).

\section{Acknowledgements}

The authors gratefully acknowledge the funding provided by the NSF ATE Award \# 1700674, MANEUVER: Manufacturing Education Using Virtual Environment Resources.

\section{References}

BLS, United States Bureau of Labor Statistics. (2014). Employment Projections: 2014-24 [occupation.XLSX]. Retrieved from http://www.bls.gov/emp/ind-occ-matrix/occupation.XLSX. 
Chandramouli, M. \& Heffron, J. (2015) March. A Desktop VR-based HCI framework for programming instruction. In Integrated STEM Education Conference (ISEC), 2015 IEEE (pp. 129-134). IEEE.

Chandramouli, M., Zahraee, M. and Winer C. (2014). A fun-learning approach to programming: An adaptive Virtual Reality (VR) platform to teach programming to engineering students. IEEE International Conference on Electro/Information Technology (EIT), Milwaukee, WI, USA. 581586.

Deloitte Review (2016). 2016 Global Manufacturing Competitiveness Index. Retrieved from http://www2.deloitte.com/global/en/pages/manufacturing/articles/globalmanufacturingcompetitiveness-index.html.

Dias, P., Sousa, T., Parracho, J., Cardoso, I., Monteiro, A., \& Santos, B. S. (2014). Student projects involving novel interaction with large displays. IEEE computer graphics and applications, 34(2), 80-86.

Digital Manufacturing (2016) Source:. Retrieved 4 October 2016 from http://searchmanufacturingerp.techtarget.com/definition/digital-manufacturing.

Jen, Y. H., Taha, Z., \& Vui, L. J. (2008). Vr-based robot programming and simulation system for an industrial robot. International Journal of Industrial Engineering: Theory, Applications and Practice, 15(3), 314-322.

Jin, G. and Nakayama, S. (2013). Experiential Learning through Virtual Reality: Safety Instruction for Engineering Technology Students. Journal of Engineering Technology 30(2), 1421. ISSN: 0747-9664.

Toth, E. E., Ludvico, L. R., \& Morrow, B. L. (2014). Blended inquiry with hands-on and virtual laboratories: the role of perceptual features during knowledge construction. Interactive Learning Environments, 22(5), 614-630.

Research and Markets (2015). DM Market in the US 2015-2019. Retrieved from http://www.researchandmarkets.com/research/cj3ggm/dm_market_in_the.

Wohlers, T. T. (2001). Wohlers Report 2001: Rapid Prototyping and Tooling State of the Industruy: Annual Worldwide Progress Report. Wohlers associates. 